\title{
Design Implementation of Distributed Stand-along Type IoT Universal Integration Platform for Information Services
}

\author{
Jin-Man Heo ${ }^{1}$ and Yang-Keun Park ${ }^{2}$ \\ ${ }^{1,2}$ Department of Research and Development, ITWORKERS Co. Ltd. \\ ${ }^{1,2}$ Korea National University of Transportation, Korea \\ ceo@shinetec.co.kr,yesok@ut.ac.kr
}

\begin{abstract}
This paper is relevant to how each databases connect to one center platform, making the best use of keeping current service structure of Stand-along server system for providing convenience to users, reduction of cost to developers because necessities of developing Stand-along server system which can integrate processing IoT sensor's information have been increased. Also, in the structure of Universal Integration Platform, it suggests the way between of base station to apply GPS system and the distance cognition way of user to recognize distance information of each users and also suggests solution plans and problems that may have possible to occur in the proposal methodology.
\end{abstract}

Keywords: IoT, Universal integration platform, GPS, NFC, BLE, Zigbee, LoRa

\section{Introduction}

Internet of Things(IoT) is called as technology or environment which can exchange data in a live attaching the sensor to some products.[1] Surely, the thing connected to internet in the current can be often seen around but Internet of Things(IoT) has institution of spread concept which is the same as above thing. So far equipments connected the internet have exchanged information as condition under involved in the operation by human, when IoT age comes to realize, it can be communicated without human taking or giving information Bluetooth or short-range wireless technology, sensor data technology, network technology can be covered by helping their own autonomous communication.

Recently, Google puts out IoT products already which hit the market such as Google glass or 'Pureband of Nike', home appliances with NFC is one of examples which are realized by IoT. Scale of the IoT products has continuously increased so that Gartner (Gartner, Inc), Global IT Advisory Organization, prospects that IoT product will attain 64 hundred million in 2016 which is increased $30 \%$ more than in 2015, and 208 hundred million in2020.[2]

Below graph shows that Gartner, Inc announced IoT semiconductor of electronic equipment profits in 2014 


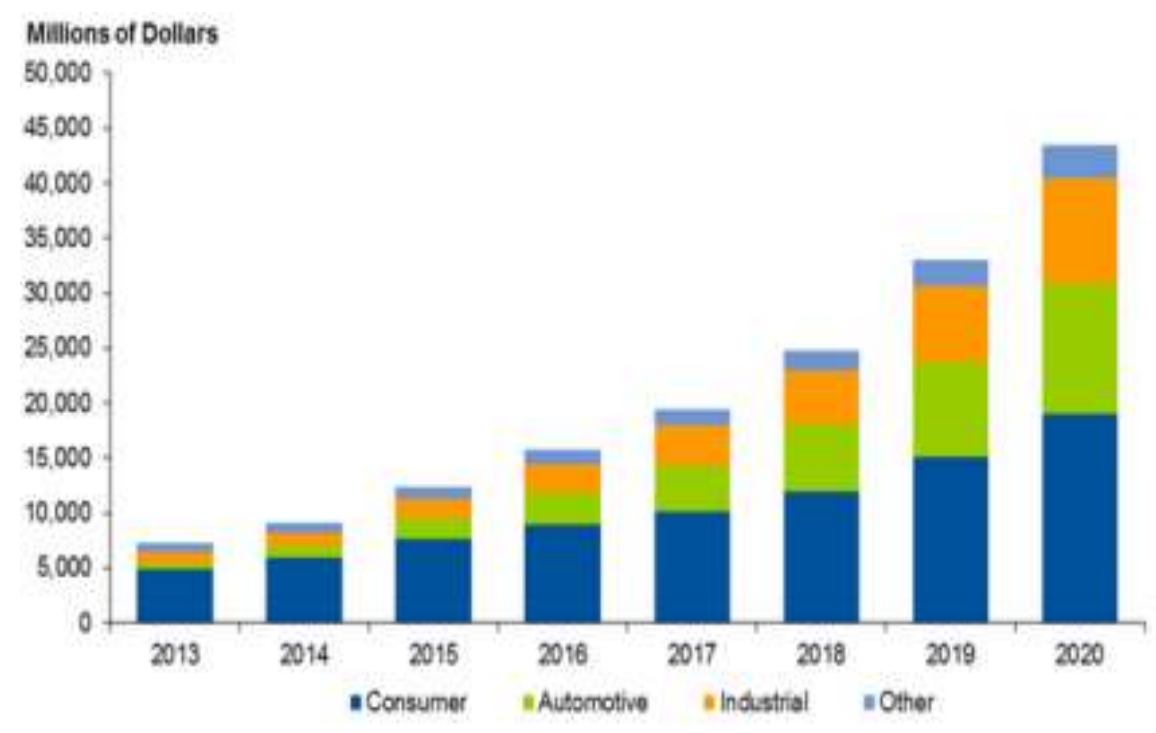

Figure 1. Anticipation of IoT Semiconductor of Electronic Equipment Profits

Following this trend, recently various types of IoT sensor have their own application and database but users have trouble with inconveniences because of numerous sensors and application.[3]-[13]

In this paper for solve the inconveniences it suggests integration platform to integrate Information services of various IoT sensors. It also proposed user's interface based on the location of user receiving the signal of each sensors in the base station and GPS hunting method to connect information which has correct user location and so on in the integration platform. Furthermore in the process of connecting the information it suggests to take methods of dealing which regards of signal strength of sensor and solving overlap problem.

People build the conversation with interesting thing so called topic when they talk to someone. Objects also need to have common topics. Temperature, humidity, heat, even ultrasonic waves they have communicating with the objects that have a type. However, they need more additional technologies when reporting among objects. It needs channel to be able to exchange the communication and that is languages to use between objects commonly.

That's what called 'Sensing technology', 'Radio wire integration', 'Network infra', 'IoT service interface technology' so on.

\section{Design Universal Integration Platform}

Figure 2 shows sort of IoT device and relationship of between objects physically. 


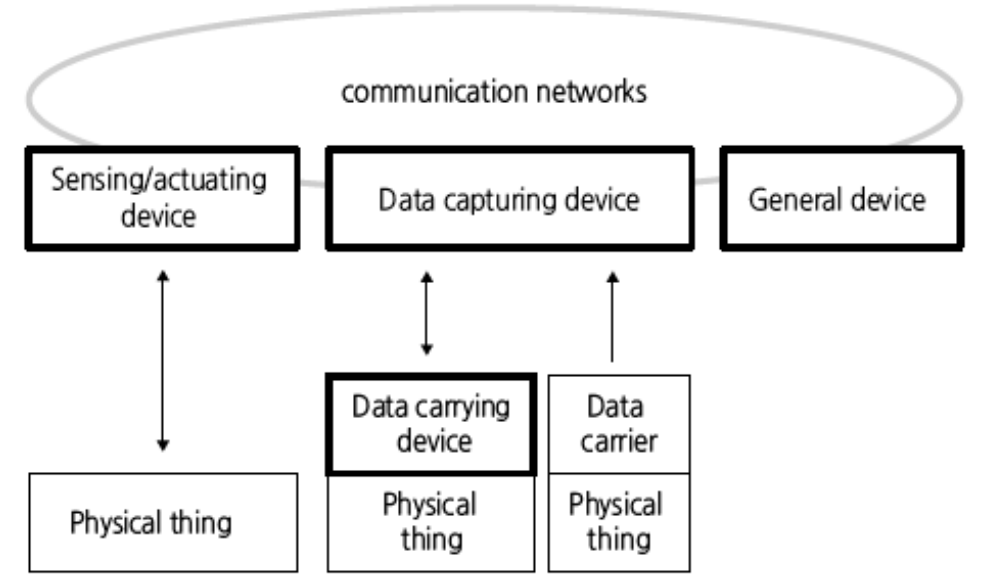

\section{Figure 2. Relationship of between Object Things and loT Device}

Minimum requirements of IoT which device has to is communication function, and it has 4 parts.

First, devices attached on objects such as Smart phone are the part that connects physical objects indirectly to communications network Second, distinguished devices such as data acquisition device attached on objects can record or read the information of physical objects with communication. Third, The devices such as all sorts of sensor or actuator can notice or measure information related to around environment. Forth, devices such as integration device have communication skill and information-processing capacity.

IoT sensors are much various, for example, NFC, RFID, QR code, Bluetooth, Beacon and have diversity in a dimension 'Spec' such as recognition distance or labeling. As a variety of parts, developers and companies/ develop various applications( Google Glass of google, Pure Band of Nike), that have their own contents and server itself. to connect each IoT sensors and objects. Developers and companies invest to spending themselves to many times and efforts to build application and server. Also users who have this application have to install several applications that are linked for receive various sensor information because it is not standardized. and that's what the paper has point of. If the each application systems not only stand as it is but connect each servers and if they connect only to developer and companies system agent, users can access to the ones which ask to install only one integration platform app.

Using many IoT sensors's cognition method or recognition distances but not changing, through integral application it can access to center Hub(integrated processing Hub) so that it use the way to get into each of substructure applications. By these system users don't need to install many cognition applications of IoT, only one leads members signed up in the system to access.

In other words, It is the point in this study that suppliers keep their system stay, which makes consumers more comfortable to access to the application.

Most important thing in this paper is to exchange information each other, keeping current system for all it's worth.

5 main IoT issues in this study which must include required to belows.

First is service which include convenience and management of users. Second is to utilize supply easy contents and device. Contents needs to connect autonomously with agent because it is placed in the existing server. Third is that sensors based various sort of sensor use standards protocol so that it needs to connect and recognize. Forth is expandability about automatic coupling and terminator devices. Whenever user's devices can add and it should be able to manage the contents if they want.

The below Figure 3 shows Intel Integration Platform Structure. 


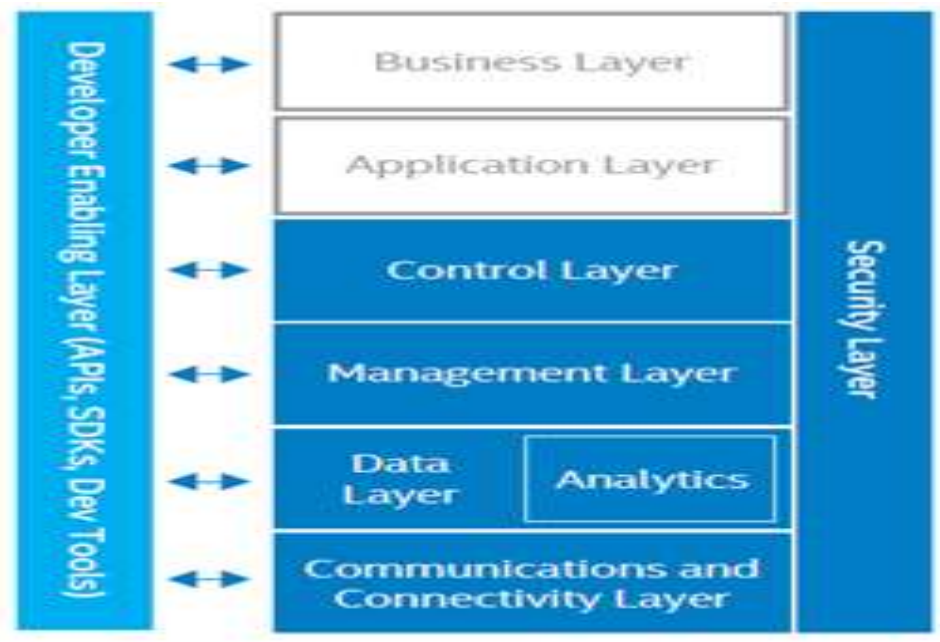

Figure 3. Intel Integration Platform Structure

The above figure is layered Intel platform structure. In there, the white part is user layer, the dark blue part is main action layer which exists Control Layer, Management Layer, Data(Analytics) Layer, and Communications and Connectivity Layer. In the last, the light blue part is the layer for developers which includes applications or development tools.

In this figure, developer layer and the others have constitutions which draws doubleway interaction, which means to respond or have feedback about several layers. Intel commercializes Cloud Service based on this structure. In case of the integration platform suggested in this paper, it provides a differentiated point that many developers or companies install connection programs called agent of each server to use the contents matched with MAC dress sensor, keeping database. Intel integration platform has different thing to log in lots of devices which get on the Cloud through gateway device.

In other words, in the Intel's Cloud service, they use the gateway device but in this paper there is the concept of using Cloud through agent program.

If it is utilized, not only smart phone but computer or another smart devices also can do communicating and more exchanging information as IoT sensor.

However, if Cloud services are utilized, it needs structured Infra. Even if the compatibility is well functioning, a perfect compatibility between A and B get limitation.

In this study, though there is no structure of huge Infra, between users and companies have no communication problems whether to not be able to convert because it connects only one integration platform for exchanging information with installing agent to database in the small and medium-sized businesses.

\subsection{Flow of Data and Connection Algorithm of Universal Integration Platform}

Connection algorithm of universal integration platform is constituted 3 steps largely. (Step 1) Install integration platform application in the user's smart device. if the smart devices are not installed, it supplies autonomous install function through NFC sensor and so on. (Step 2) If user access near to the sensor with smart device, it can communicate with matched service server agent searching service what they want cognizing sensor ID in the application. (Step 3) The recognized sensor for user gives the contents and the information of smart device through agent SW of service server.

This method is very simple principle and it takes advantage to access all information utilizing only one standard platform application without installation of the extra service application. Users can save their times and efforts with only one practicable application. The below picture shows process of accessing 3 steps which explain in the above as flowchart. 


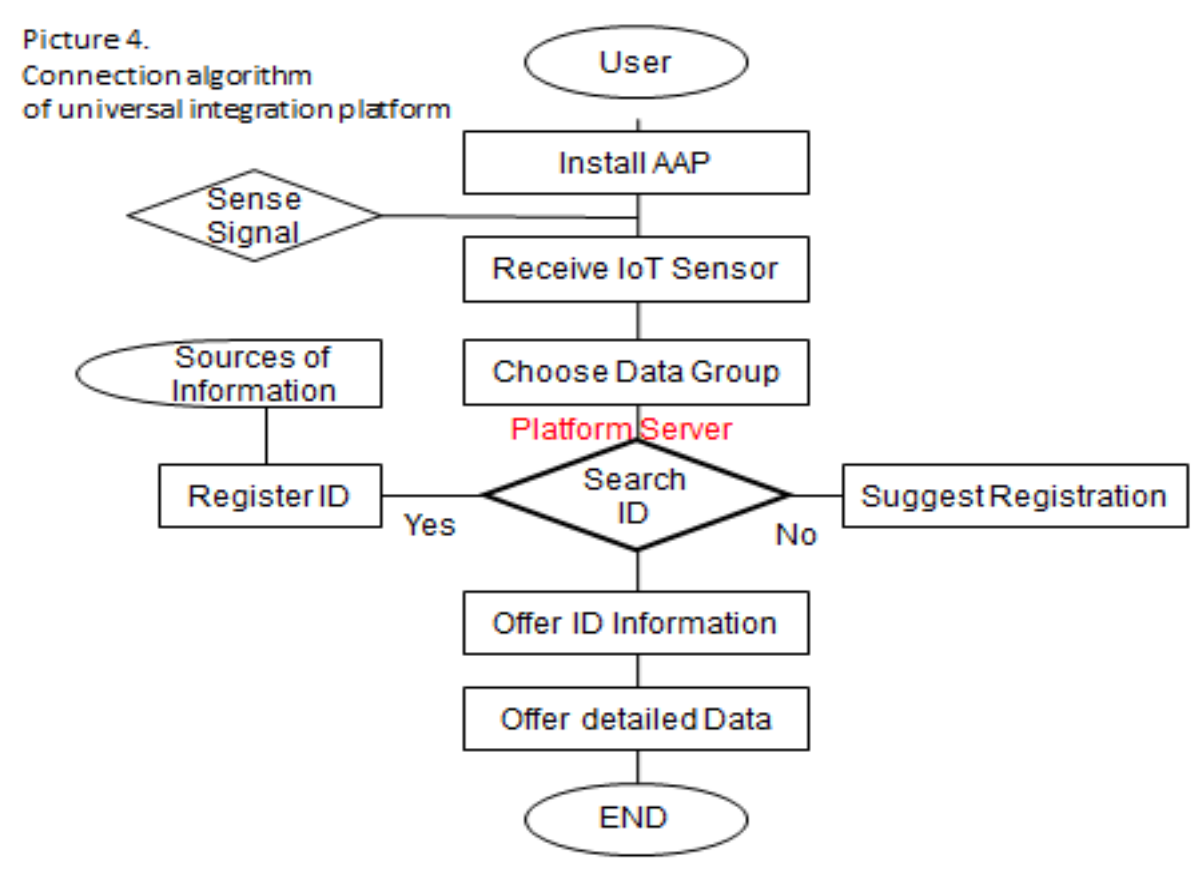

Figure 4. Connection Algorithm of Universal Integration Platform

Universal integration platform does not work all of things by itself. It is simply support to access to more easily as supporter. If all of existing information save to one application and $\mathrm{DB}$, the handling capacity of data and storage will sharply increase to save all of database information of service and eventually it wouldn't be saved. In integral platform, therefore, the structure which has to connect each service database functions only connecting each companies and users as setting up a link to minimize the handling capacity of platform.

\subsection{Platform's Class of Structure and Connection}

When user needs to communicates accessing database made by developer or company, it is needed to connect to hierarchy structure of universal integration platform that is, it is carried out, passing each class.

First, communication and connection class. It is physical class that makes it happen to communicate with multi-protocol data among units not only gateway but also network, database. Also, it controls data insertion and device control function in the 5 IoT issues above. Second, Data and Analysis hierarchy.

This class controls the function which analyzes data such as database, universal integration platform, each sensor units, or physical address between devices. Furthermore it controls data between unit device and database. Third, Management class. This class makes possible to support information of device and detection of autonomous device. Also, this class is related to database of each companies closely because universal integration platform dose have only keyword so that it can connect to user to user so it is essential for database to have this class. Fourth, Control class. This class has motion with management class together including control devices of application and policies. It is not upper class than management class but co-operative relationship. Fifth, Supplementation class. This class includes 4 classes that explain above. Database made by each companies and developers includes each of supplementation's protocols so does universal integration platform. Therefore, it makes supplementation protocols compatible complementally not to be off the 5 issue of IoT points. 


\subsection{Location Sensing Algorithm Based on GPS and Base Station}

For make user easy to access Single service platform connecting the integral application, this paper adopt system of base station. In the current smart devices, there are equipped with GPS so that it measures the distance of between user and the place where IoT sensor exists, which called receiver. If there are receiver A and B around user, the GPS technology recognizes the closer one of them. As a result, user can have access to closest application of receiver automatically and more easily without finding application of many receivers.

What should we do when GPS turned off? To solve this problem, It suggests conception of understanding Position information based on base location. To communicate between base location and land mobile station set by many communication companies or by relay in mobile relay station, the base station sets in land though there are many base locations based on above things named radio station that is fixed and even GPS get turned off it can fully recognize distance of between user and receiver.

However, What recognize distance information between user and receiver can be told less efficient in terms of cost due to necessity of separate transmit with base location.

What we call 'IoT environment' is that devices composed by many IoT units service to user through communication or connection each other without any interferences or cognition. To compose such IoT environment, devices have to get the Internet and web protocol. However in this paper, for increasing convenience of users and small infra there are not including web protocol which is made by each company and developer. That's why GPS and base station are used. As recognizing GPS and base station for user's distance, it allow this paper to accord with its object with making not to touch the database built by each companies and developers.

\subsection{Receiver Data Processing of Universal Integration Platform}

If user comes in the range of GPS or base station, the representative sensor of user which recognizes only distance with user shows making sign information of receiver to smart device of user.

If user want to browse receiver information, universal integration platform connects sensor ID which is inherent ID to each platforms as keyword. In this way user can browse the information of each receivers. How to find the information or share at that time, there are Sequential elimination rule, Arrange type, and Detection number system.

Sequential elimination rule is the way to recognize user's signal first and then dose user's information. Arrange type is the way to be able to choose in person to make user's information a content. Last is Detection number system which has the way to understand one of the most overlapped signals during that time, as overlapping some distances by GPS and base station. In other words, Detection number system rather than Sequential elimination rule, Arrange type is able to be the way to recognize universal integration platform to GPS and base station.

The below picture shows Sequential elimination rule, Arrange type, and Detection number system in Figure 5. 

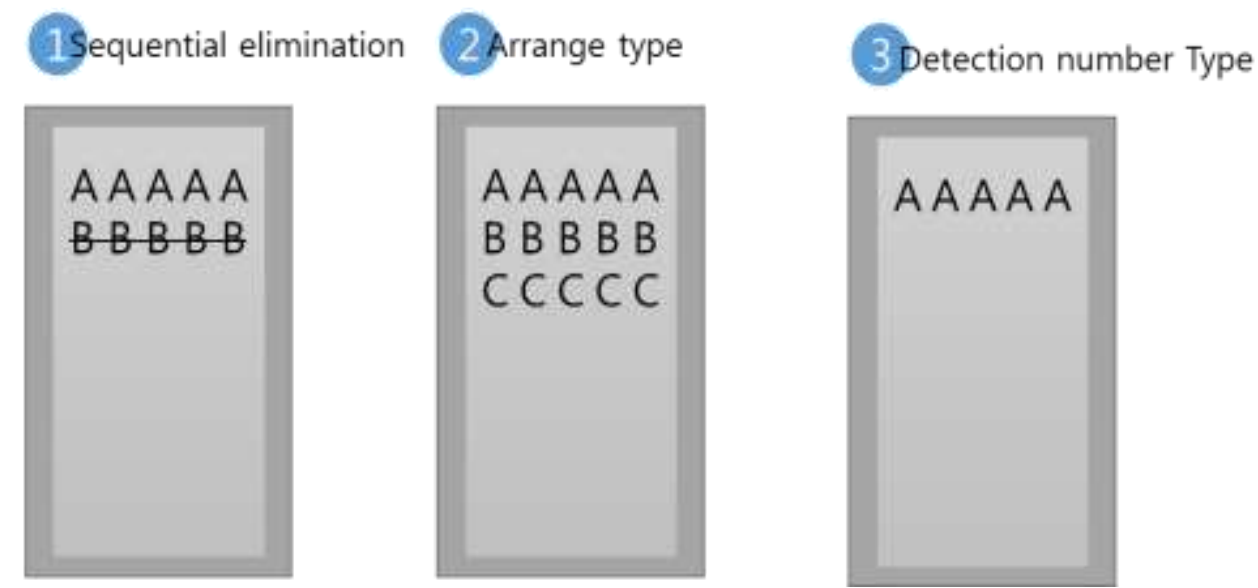

\section{Picture 5. Way to Share or Find Information}

\subsection{Access Protocol Data Base}

It explains how user does access the data base which made by companies or developers. When user get the IoT sensor signal, it recognizes the unique MAC address of sensor and starts to search through this. The standard platform which takes all MAC address of sensor recognizes itself so that it can perform a role of letting the relevant organization agent know about which organization is. The agent performs a role of finding contents accorded with the MAC address of sensor in the relevant organization and giving back information of contents on the smart phone. In other words, agent is fast for finding only in the data base of relevant organization not finding contents in all application service. Platform allows to know where contents are but the role of passing the contents to smart phone is a part of agent which carries out in the application server without worry of traffic and the part is a concept to give to manage all of application service in the standard platform.

\section{Conclusion}

This paper is to develop and realize the structure which can handle IoT sensor signals with universal integration platform.

In this paper, various IoT signals such as Bluetooth, NFC, and Beacon consider to be connected with a series of universal integration platform and make it effect on capacity, expense, and convenience of server. Also, doing figure out of user suggest the way to transmitting through GPS and base station and universal integration platform suggest the way to cut down expenditure and capacity using only sensor ID (inherent MAC address) in the each receiver's DB. The most characteristic thing in this paper is that each sensor which is existing approach each different application and data base to work each service which is made for their own sensor primely so that in universal integration platform it can be accepted and suggest the way to connect the relevant contents through inherent address of sensor install the agent in what offers application service to server.

\section{Acknowledgments}

This work (Grants No.C0330539) was supported by Business for Cooperative R\&D between Industry, Academy, and Research Institute funded Korea Small and Medium Business Administration in 2015. 
[1] IoT(Internet of Things). Terminology of IT. Blotter by Lee Ji Young (2013.11.07.)

[2] Gartner Inc. Press Release, (2014).

[3] Y. Kim, Y. Jeon and I. Chong, "Device Objectification and Orchestration Mechanism for IoT Intelligent Service", kics.2013.38C.1.19, (2013).

[4] Ministry of Science, ICT and Future Planning, ".IoT basic paln”, (2014).

[5] Korea Communications Commission, "Korea Communications Future Service Strategy", (2010).

[6] Korea Communications Commission, "Study System of Korea Communications Convergence Future Strategy", (2010).

[7] Korea Communications Commission, "No.48 Korea Communications Commission Conference", (2009).

[8] Y. B. Seong and K. S. Min, "SDN based IoT Platform for Big Data Processing", Korea Telecom Society Winter Synthesis Conference, (2016).

[9] E. J. Am, K. N. Su, K. J. Gil, P. T. Jun, K. H. Yong and P. C. Sik, "IoT device products and technology trends", Electronics and Telecommunications Research Institute.2014.12.31C, (2014).

[10] J. Chungmin and N. Hyungjin, "A Study of Research Trend about Internet of Things", Information Policy, vol. 22, no. 3, (2015).

[11] Cisco, "Visual Networking Index", (2011).

[12] ITU-T, "Overview of the Internet of Things," Y.2060, (2012).

[13] Intel. "The Intel IoT Platform", (2015).

\section{Authors}
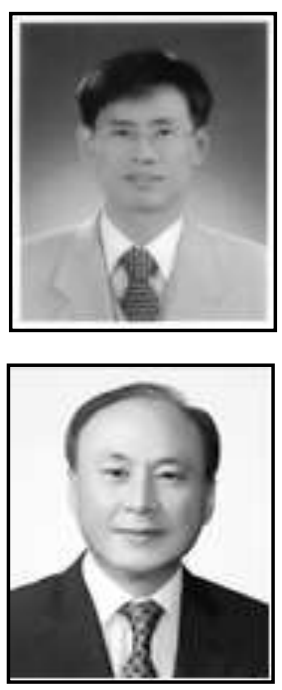

Jin Man Heo, jin Man Heo curricular his Ph.D in Computer Science from University of Sun-moon in 2010. He is IT Scientists and Researcher, Department of Research and Development, ITWORKERS Co. Ltd., from 2014 to 2016.

Yang Keun Park, Yang Keun Park received his Ph.D in Administration Business from University of Ho-seo in 1987. He is a Professor in Korea National University of Transportation College of Social Science from 2012 to 2016. 